\title{
Guaranteeing Method for the Stability of Cluster Structure Formed by Autonomous Decentralized Clustering Mechanism
}

\author{
Ryo Hamamoto ${ }^{1}$, Chisa Takano ${ }^{1}$, Kenji Ishida ${ }^{1}$, and Masaki Aida ${ }^{2}$ \\ ${ }^{1}$ Graduate School of Information Sciences, Hiroshima City University, Hiroshima-shi, Hiroshima, 731-3194 Japan \\ ${ }^{2}$ Graduate School of System Design, Tokyo Metropolitan University, Hino-shi, 191-0065 Japan \\ Email: ryo@net.info; \{takano, ishida\}@hiroshima-cu.ac.jp; aida@tmu.ac.jp
}

\begin{abstract}
Mobile ad hoc networks (MANETs) can be configured by mobile terminals without any network infrastructure such as Access Points (APs), and they are expected to be a useful tool during serious disasters such as a large earthquake. Many studies have concentrated on clustering methods that aim at power saving and loadbalancing in MANETs. For the clustering mechanism of a MANET, we have proposed an autonomous decentralized structure formation method based on the local interaction of terminals and have used this method to create an autonomous decentralized clustering of MANETs. However, the problem of our proposed clustering method is that the number of clusters decreases with temporal evolution. The power saving and the load balancing by the hierarchical management of the network cannot be performed by this problem. This paper proposes a method which maintains the number of clusters (guarantees stability).
\end{abstract}

Index Terms-Autonomous decentralized control, localaction theory, ad hoc network, clustering

\section{INTRODUCTION}

When almost all network infrastructure is destroyed by a serious disaster, it is necessary to create an environment in which the remaining devices can operate effectively and distribute information quickly in order to support smooth disaster restoration activities. Mobile ad hoc networks (MANETs) [1] have received significant attention in recent years for such an emergency situation. MANETs directly connect network terminals to each other without network infrastructure such as wireless LAN access points. At the time of a disaster, reducing the power consumption of terminals is very important. Many studies have concentrated on clustering methods that aim at power saving and load balancing in MANETs [2]. Here, clustering methods divide the network to multiple subnetworks (clusters) and realize hierarchical management for the inter-cluster and the intra-cluster. Power saving and load balancing are crucial because they make it possible to reduce the power consumption of each

Manuscript received January 11, 2015; revised August 19, 2015.

This research was partly supported by JSPS KAKENHI Grant Numbers 26280032, 15K00431, and Project Research Grants from the Graduate School of Information Sciences, Hiroshima City University. Corresponding author email: ryo@net.info.hiroshima-cu.ac.jp doi:10.12720/jcm.10.8.562-571. node and extend the life of the network after a disaster. Some clustering methods realize both by using metrics such as each node's battery reserves [3] and performance [4]. Generally, global network state information is required to optimize the cluster structure. However, in MANETs, information exchange is structurally limited, and each node needs to execute traffic control, path control, and network resource management using only local information.

Many studies have targeted the clustering algorithm [5]-[7]. However, since their algorithms require nonlocal information, they are not "strictly" autonomous decentralized algorithms. The common basic attribute demanded from these algorithms in addition to locality is adaptability to the network environment. Each node has its specific situation that influences the formation of clusters. So, it is preferable for the clustering mechanism to take the situations of individual nodes into consideration. [8], [9] can configure clusters through only local information by the well-known bio-inspired approach that uses Turing patterns formed by reactiondiffusion equations. However, this method cannot yield cluster structures that reflect the characteristics of the given environmental conditions (e.g. the distribution of the residual battery power of terminals, the position of power supplies, or the node degree of mobile terminals) [10]. Moreover, because this method uses seven parameters, it is difficult to identify the appropriate values for these multiple parameters.

[10]-[12] have already proposed a framework of autonomous decentralized control based on local interaction as a novel control mechanism. This framework is based on the relationship between the local interaction and the solution provided by a partial differential equation. As a specific example, [10] have proposed the autonomous decentralized formation of structures with finite spatial size and showed that proposal's applicability to autonomous decentralized clustering in ad hoc networks. The clustering method of [10] allows the nodes to act flexibly in a manner based only on the information each individual node is aware of, i.e. its individual situation, and it can yield cluster structures that reflect the characteristics of the given environmental conditions. In [13], the method of [10] can 
configure clusters faster than an existing method [8] by a factor of 10 or more. This means that communication can be recovered more quickly by the method of [10].

However, the problem of [10] is that the number of clusters decreases with temporal evolution. The power saving and the load balancing by the hierarchical management of the network cannot be performed by this problem. This paper proposes a method which maintains the number of clusters (guarantees stability). So, our aim is to maintain the hierarchical network configuration created by clusters. Our study focuses on the hierarchical network configuration that is the preliminary study needed to realize the real communication considering data transfer by network protocols.

This paper consists of the following sections. In Sec. II, we present the framework of our proposed autonomous decentralized structure formation method, and we discuss the issues for this method. In Sec. III, we propose a method of guaranteeing the stability by preserving the history of the distribution. We evaluate the characteristics of the proposed method in Sec. IV, and Sec. V presents the concluding remarks.

\section{Clustering Method Based on Autonomous DECENTRALIZED STRUCTURE FORMATION}

This section overviews the autonomous decentralized structure formation proposal [10] that uses the diffusion and the drift (back diffusion). Moreover, we describe the problem of our structure formation method that is applied to clustering in MANET.

\section{A. Overview of the Autonomous Decentralized Structureformation Method}

First, we introduce the autonomous decentralized structure formation method for a one-dimensional network model to understand the behavior of our proposal intuitively. Let the density function (density distribution) of a certain quantity at time $t$ and position $x$ be $q(x, t)$. The initial value of $q(x, 0)$ can be considered as the metric, for example, the residual battery life of each node in an MANET. Local behavior corresponds to changing the value of $q(x, t)$ at each point, $x$, by controlling the information exchange between adjacent nodes. In the autonomous decentralized structure formation, the flow $J(x, t)$ (the operation rule that changes the value of $q(x, t))$ is expressed as

$$
J(x, t)=-c f(x, t) q(x, t)-c \sigma^{2} \frac{\partial}{\partial x} q(x, t)
$$

where the first and the second terms denote the drift and diffusion terms, respectively. The temporal evolution of the distribution $q(x, t)$ that corresponds to this change is given by

$$
\frac{\partial}{\partial t} q(x, t)=c\left(\frac{\partial}{\partial x} f(x, t)+\sigma^{2} \frac{\partial^{2}}{\partial x^{2}}\right) q(x, t)
$$

$c(>0)$ denotes the rate of the temporal evolution of $q(x, t)$ and $\sigma^{2}$ denotes the variance of the normal distribution that is converged on. $J(x, t)$ represents the amount of spatial movement of $q(x, t)$; note that the total amount of $q(x, t)$ does not change over time. Equation (2) is a second-order differential equation. Therefore, this operation rule can be determined by interaction with the adjacent nodes.

The introduction of $f(x, t)$ eliminates the need to set a coordinate system in the network. As a more intuitive explanation, we consider the potential function $\Phi(x, t)$ instead of the function $f(x, t)$ :

$$
f(x, t)=-\frac{\partial \Phi(x, t)}{\partial x}
$$

Choosing $\Phi(x, t)$ appropriately yields autonomous decentralized control that does not depend on a coordinate system. We consider how to determine the drift term from $q(x, t)$ at each point $x$. Because $\Phi(x, t)$ should result in maintaining the distribution within a certain finite spatial size, contrary to the effect of diffusion, $\Phi(x, t)$ is, after a discrete time $\Delta t$, given by

$$
\Phi(x, t+\Delta t)=-\left(q(x, t)-\gamma \frac{\partial^{2} q(x, t)}{\partial x^{2}} \Delta t\right)
$$

where $\gamma>0$, and $\Phi(x, t)$ is periodically renewed at intervals of $\Delta t$. This equation is obtained by the sign inversion of the space derivative term in the diffusion equation. Note that in Eq. (4), we use a periodical time with interval $\Delta t$ instead of $\mathrm{d} t$. This is because the effect of the second term vanishes in the limit that $\mathrm{d} t$ approaches 0 [10].

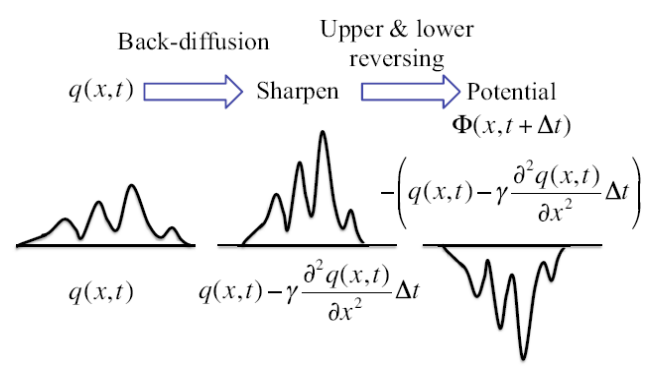

Fig. 1. Determining $\Phi(x, t)$ according to back diffusion.

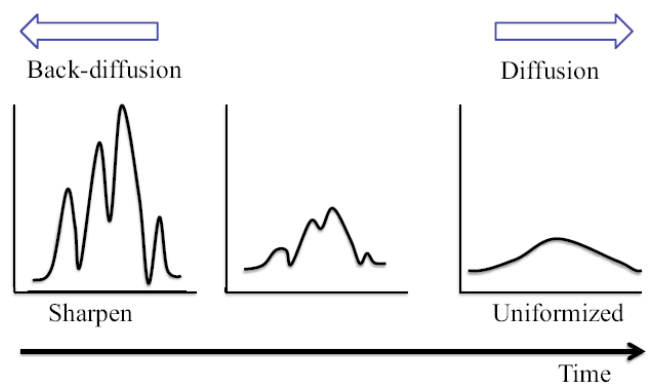

Fig. 2. Diffusion and back diffusion.

The method of generating the potential $\Phi(x, t+\Delta t)$ by using $q(x, t)$ is shown in Fig. 1 . The meaning of this figure is expressed as follows:

- We let the time progression of the diffusion phenomenon with diffusion coefficient be reversed (back diffusion). 
- Next, we reverse the distribution (up and down) and regard the completed distribution as the potential.

Because of the effect of the drift term, including the potential, the peak of $q(x, t)$ is emphasized, and the shape of the distribution is sharpened (Fig. 2). The effect of the diffusion term, on the other hand, is to flatten the distribution.

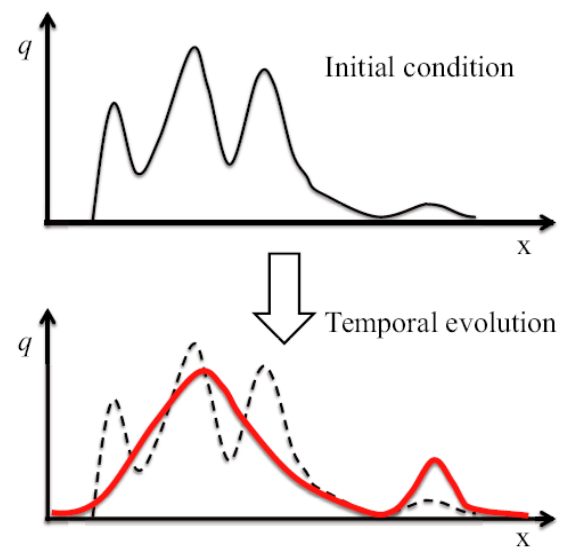

Fig. 3. The effect of the back diffusion in one-dimension model.

Here, we explain the characteristics of the proposed method using by Fig. 3. Let us consider the initial condition on an one-dimension model in which there are two or more peaks on the left, and there is one smallscale peak in the right. In areas that have multiple peaks, the peaks are integrated autonomously to form one cluster. On the other hand, when there is no peak in the surrounding, the peak is emphasized even if it is not a powerful peak. This effect derives from the back diffusion, and our proposal adopts back diffusion to form the cluster even for a remote weak peak.
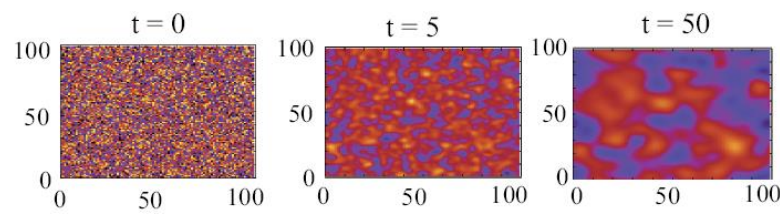

Fig. 4. Cluster structure formation by our proposed scheme (initial condition, after 5,50$)$.

Fig. 4 shows an example of the structure with a finite spatial size that can be formed by balancing one effect against the other. In Fig. 4, the peak of $q(x, t)$ is the representative node of the cluster and the minimum value of the distribution is the boundary of the cluster.

Moreover, [10] showed that our approach can be applied to an arbitrary network instead of a onedimensional network, because the diffusion and back diffusion can be defined based on just the state of the node and the adjacent nodes. Let us describe the local action rule in the network concretely. First, the set of nodes that are adjacent to node $i$ (set of nodes that are linked to node $i$ ) is defined as $N i$. In addition, we discretize time, and set the interval time of the autonomous control to be $\Delta t$.

In the following, we describe the action rule for spatial discretization that corresponds to nodes in the network and time discretization that corresponds to control timing. The distribution $q_{i}\left(t_{k}\right)$ at time $t_{k}(:=k \times \Delta t)$ at node $i$ changes the next time (after $\Delta t$ passes) as follows:

$$
q_{i}\left(t_{k+1}\right)=q_{i}\left(t_{k}\right)-c \Delta t \sum_{j \in N_{i}}\left(J_{i, j}^{\mathrm{drift}}\left(t_{k}\right)+J_{i, j}^{\mathrm{diff}}\left(t_{k}\right)\right)
$$

where $J_{i, j}^{\text {drift }}(t)$ and $J_{i, j}^{\text {diff }}(t)$ are the variations created by the drift effect and the diffusion effect within each unit time, respectively. $J_{i, j}^{\text {drift }}(t)$ and $J_{i, j}^{\text {diff }}(t)$ satisfy the following equations:

$$
\begin{aligned}
J_{i, j}^{\mathrm{drift}}\left(t_{k}\right) & :=\left\{\begin{array}{cc}
f_{i, j}\left(t_{k}\right) q_{i}\left(t_{k}\right), & \left(f_{i, j}\left(t_{k}\right)>0\right), \\
-f_{j, i}\left(t_{k}\right) q_{j}\left(t_{k}\right), & \left(f_{j, i}\left(t_{k}\right)>0\right),
\end{array}\right. \\
f_{i, j}\left(t_{k}\right) & :=-\left(\Phi_{j}\left(t_{k}\right)-\Phi_{i}\left(t_{k}\right)\right), \\
J_{i, j}^{\mathrm{diff}}\left(t_{k}\right) & :=-\sigma^{2}\left(q_{j}\left(t_{k}\right)-q_{i}\left(t_{k}\right)\right) .
\end{aligned}
$$

Due to the drift effect, the distribution moves in direction $i \rightarrow j(j \rightarrow i)$ in the case of $f_{i, j}\left(t_{k}\right)>0$ $\left(f_{i, j}\left(t_{k}\right)<0\right)$. Here, equation $f_{i, j}\left(t_{k}\right)=-f_{j, i}\left(t_{k}\right)$ holds. The variation is proportional to the product of the velocity of the drift $f_{i, j}\left(t_{k}\right)\left(f_{j, i}\left(t_{k}\right)\right)$ and $q_{i}\left(t_{k}\right)\left(q_{j}\left(t_{k}\right)\right)$ in node $i(j)$. The above description is formalized by Eq. (6). The variation due to the diffusion effect is proportional to the gradient of the distribution in Eq. (8).

Next, we explain how to determine the potential $\Phi_{i}\left(t_{k}\right)$ that is related to drift. The potential value $\Phi_{i}\left(t_{k+1}\right)$ of node $i$ at time $t_{k^{+1}}$ is decided by the value of the distribution $q_{i}\left(t_{k}\right)$ and the back diffusion of $q_{i}\left(t_{k}\right)$ as follows:

$$
\Phi_{i}\left(t_{k+1}\right)=-\left(q_{i}\left(t_{k}\right)-\gamma \Delta t \sum_{j \in N_{i}}\left(J_{i, j}^{\mathrm{back}}\left(t_{k}\right)-J_{j, i}^{\mathrm{back}}\left(t_{k}\right)\right)\right)(9)
$$

where $J_{i, j}^{\text {back }}\left(t_{k}\right)$ is generated by the back diffusion of $q_{i}\left(t_{k}\right)$, and is the variation in a unit time period in the direction of node $i \rightarrow j$. The variation $J_{i, j}^{\text {back }}\left(t_{k}\right)$ is given by

$$
\begin{aligned}
& J_{i, j}^{\text {back }}\left(t_{k}\right)=\left\{\begin{array}{cc}
q_{j}\left(t_{k}\right)-q_{i}\left(t_{k}\right), & \left(\Delta q_{i}^{\max }\left(t_{k}\right)\right. \\
\left.=q_{j}\left(t_{k}\right)-q_{i}\left(t_{k}\right)\right), & (10, \\
0, & \text { (otherwise) }
\end{array}\right. \\
& \Delta q_{i}^{\max }\left(t_{k}\right):=\max \left(\max _{j \in N_{i}}\left(q_{j}\left(t_{k}\right)-q_{i}\left(t_{k}\right)\right), 0\right)
\end{aligned}
$$

$\Delta q_{i}^{\max }\left(t_{k}\right)$ is the difference between the distribution value of node $i$ and the distribution value of the adjacent node $j$ in the direction of the steepest ascent from node $i$. In the above-mentioned action rule for discretization in the network, local interaction is guaranteed because the summations for nodes $j \in N_{i}$ in the above equations involve only the nodes adjacent to node $i$.

To achieve the above mentioned control, it is necessary to exchange information about the values of distribution $q_{j}\left(t_{k}\right)$ for the adjacent nodes at the interval of $\Delta t$. The complexity of this information exchange does not depend on network size because the communication range is just 
1 hop. Therefore, it is scalable against the number of nodes.

Note that, $c \sigma^{2}$ needs to satisfy Eq. (12)

$$
c \sigma^{2}<\frac{1}{d_{\max }}
$$

where $d$ max means the maximum degree of nodes in the network. If $c \sigma^{2}$ do not meet Eq. (12), $q($.) of some nodes become negative value by the diffusion effect [14].

\section{B. Problems with Applying the Proposal to Clustering}

In this section, we discuss the issues raised by applying this technology to MANETs. The autonomous decentralized structure formation proposal described in Sec. II-A forms some clusters by balancing the diffusion term (diffusion effect of the distribution) against the drift term (emphasizing effect of the peak of the distribution). If the diffusion effect is greater than the drift effect, the distribution of the formed structure is smoothed over time and the range of the distribution decays (Fig. 5). Here, the range $R(t)$ denotes the difference in the maximum value and the minimum value of the distribution for each time as below:

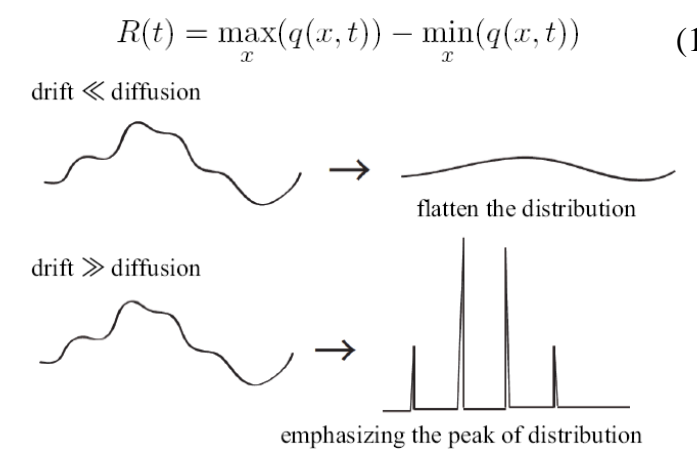

Fig. 5. Issues in parameter setting.

On the other hand, if the drift effect is stronger than the diffusion effect, the peak of the structure is emphasized, and the unevenness of initial distribution becomes excessive. Therefore, it is necessary to balance of the strengths of the diffusion and drift terms, but designing the optimum parameters is very difficult. Let us consider the situation where the diffusion effect is stronger than the drift effect. In that case, the range of the distribution decays over time (Fig. 6). Note that we evaluate the characteristics of the decay of the range in Sec. IV-A.

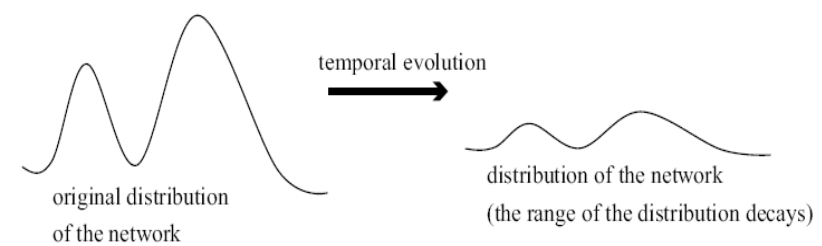

Fig. 6. Decay of the range of the distribution.

The decay characteristics cause two problems: First, the representative nodes and the boundaries of clusters cannot be determined when the range of the distribution becomes zero (complete harmonization) by the diffusion effect. In the mathematical expression, the complete harmonization practically requires infinite time; however, the range of the distribution becomes zero in finite time owing to the limits of the calculation accuracy, which means that the proposed method cannot configure the clusters. That is, the power saving and the load balancing by the hierarchical management of the network cannot be performed by this problem. Second, we should consider a situation where the distribution of one network is compared to the distribution of another network. This problem may occur in an MANET when new terminals connect or other terminals move into the area under consideration. For example, "network A" has a small range of the distribution by the diffusion effect, whereas "network B" has a large range of the distribution. Note that this situation is that the initial range of the distribution of "network $A$ " is the same as that of "network B," but the range of the distribution of "network A" decays because of a lapse of time from the cluster formation and is smaller than that of "network B." When these networks merge, "network A" will not be able to preserve its form, because their networks have the different measures for the ranges of the distributions (Fig. 7). In other words, a cluster which has large range of the distribution has a huge effect on the cluster compared to a cluster which has small range of the distribution.

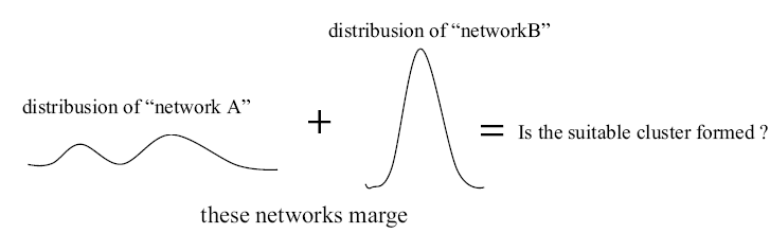

Fig. 7. Problems with dynamic network topologies.

\section{Method Guaranteeing Stability of THE Cluster STRUCTURE FOR A VECTOR PROCESS}

In this section, we discuss the requirement that the range of the distribution should satisfy in order to restrain the decay of it, and then propose a method which meets the requirement.

As mentioned in Sec. II-B, it is necessary to restrain the decay of the range caused by the time progression in order to maintain the cluster structure. This is the requirement for the range of the distribution. To show the requirement for the range of the distribution, we introduce the asymptotic stability for the range of the distribution as follows.

$$
\lim _{t \rightarrow \infty} R(t)=\alpha
$$

where $R(t)$ is defined in Eq. (13) and $\alpha$ is a positive constant. This equation means that the range of the distribution converges on a positive constant value over infinite time. Unless it is satisfied, on the other hand, $R(t)$ approaches zero with time.

Next, we explain our method which restrains the decay of the range and guarantees the asymptotic stability of the range. To guarantee the stable range of the distribution, each node has its own vector with $N$ components to keep 
the history information of the distribution. Each node has its own value, $p\left(t_{k}\right)$, that denotes the distribution (for example, battery residual power) at time $t_{k}(k=0,1, \cdots)$. $\boldsymbol{Q}\left(t_{k}\right)$ shows the distribution used for configuring the cluster structure at $t_{k}$; it is given as follows:

$$
\begin{aligned}
& \boldsymbol{Q}\left(t_{k}\right)=\left(q_{0}\left(t_{k}\right), q_{1}\left(t_{k}\right), \cdots, q_{N}\left(t_{k}\right)\right) \\
& q_{0}\left(t_{k}\right)=p\left(t_{k}\right)
\end{aligned}
$$

The vector's 0th component is set to $p\left(t_{k}\right)$. In each step, the diffusion and drift (back diffusion) operations are performed for every component of $\boldsymbol{Q}\left(t_{k}\right)$. In other words, each component of the vector is calculated by the diffusion and the back diffusion. When the operations are completed for every component of $\boldsymbol{Q}\left(t_{k}\right)$, the $n$th ( $n=0,1, \cdots, N)$ component of the vector is shifted to the $n+1$ th component in the following step (Fig. 8). Note that $N+1$ th component is discarded. If $p\left(t_{k}\right)$ does not change over time, $\boldsymbol{Q}\left(t_{k}\right)$ will not change either. A cluster is constructed by using $q^{n}\left(t_{k}\right)$ (a specified $n$ within $n=0,1, \cdots, N$ ) which each node has, and larger clusters can be configured by specifying the larger $n$.

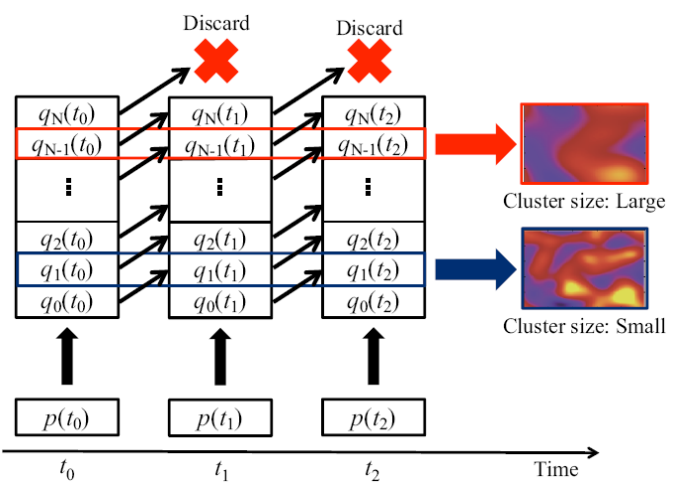

Fig. 8. Temporal evolution of $\boldsymbol{Q}\left(t_{k}\right)$ for each node.

In general clustering, it is demanded that the number of cluster heads (the representative nodes) in the network is small (in other words, the average cluster size is large) because nodes chosen as cluster heads have a heavy load due to the management of the control information for both the inter- and the intra-cluster. If the cluster size is large (the number of clusters is small), on the other hand, significant traffic occurring in bursts within the cluster causes the overload of functions for the cluster head because each cluster head attends to many member nodes in the cluster. Here, the main purpose of this paper is not to argue about the optimal cluster size. The purpose of this paper is to maintain the hierarchical network structure created by clusters. Note that our proposed method can adjust the mean number of clusters to some extent according to the network situation by only using local information exchange. The vector process of our proposed method can be simply expressed as follows: Each node keeps $N$ distribution values for each time by using the vector structure $q_{n}\left(t_{k}\right)$. The values have a hardly diffused distribution when $n$ is small and an almost completely diffused distribution when $n$ is large.
Here, $R n\left(t_{k}\right) \quad(n=0,1, \cdots, N)$ is the range of thedistribution for the $n$th component at time $t_{k}$ as follows:

$$
R_{n}(t)=\max _{x}\left(q_{n}\left(x, t_{k}\right)\right)-\min _{x}\left(q_{n}\left(x, t_{k}\right)\right)
$$

In our proposed method, if the distribution $p\left(t_{k}\right)$ (e.g. the battery capacity) does not change with time, the vector structure $\boldsymbol{Q}\left(t_{k}\right)$ that each node keeps does not change as well. That is to say, the asymptotic stability of the range in Eq. (14) is expressed as follows:

$$
R_{n}(t)=\alpha \quad\left(t \geq t_{n}\right)
$$

where $R n(t k)$ can be calculated by Eq.(17), and $t_{n}$ is the period of time used to form the $n$ components of the vector structure from nothing. Eq. (18) shows the requirement to stabilize the range of the distribution not "asymptotically" but "completely", if nodes are immovable and the initial distribution for each node does not change. When the distribution $p\left(t_{k}\right)$ becomes smaller with time, the decay of the range of the distribution cannot be stopped, but our proposed method can restrain the range decay phenomenon caused by diffusion effects. The issue for our method is that a certain time $t_{n}$ is required when creating a vector structure from nothing. The range of the distribution decays by the diffusion effect until the vector structure is formed. We will address this issue in future work. In addition, our proposed method can be applied to other clustering methods [15].

\section{EVALUATION}

In this section, we investigate the decay characteristics of the range of the distribution shown in Sec.II-B, and we evaluate the stability of the proposed method using a twodimensional lattice model and an MANET model.

\section{A. Decay Characteristics of the Range}

First, we examine the decay characteristics of the range drift effect and diffusion effect. As preliminary research, we assume a two-dimensional lattice model with $100 \times$ 100 nodes to make the space structure easy to display. The network model has a torus topology to exclude the influence of the boundary (Fig. 9). Each node has a degree of four $\left(d_{\max }=4\right)$ because each node has four neighbors, and sends a control packet to their neighbors at every step ( $\Delta t=1$ step), which is the normalized time. In this evaluation, the node sends control parameters for adjacent nodes at the same time. Moreover, each node does not move in this evaluation. Our assumed initial state of the distribution is the state where clusters have been already formed to some extent (Fig. 10). Because the purpose of the study is to investigate the range characteristics of the distribution due to the diffusion effect and the drift effect, intense unevenness of the initial state of the distribution quantity is not needed. The horizontal and vertical axes in Fig. 10 represent the coordinates, and the color of each node indicates the distribution height. 


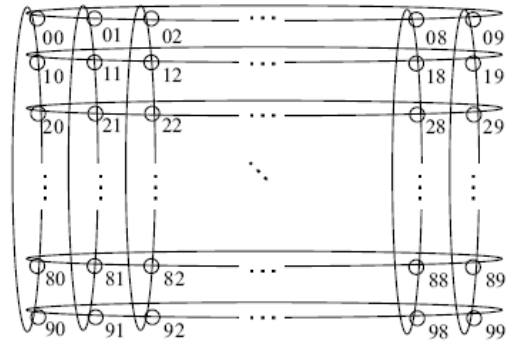

Fig. 9. Torus topology.

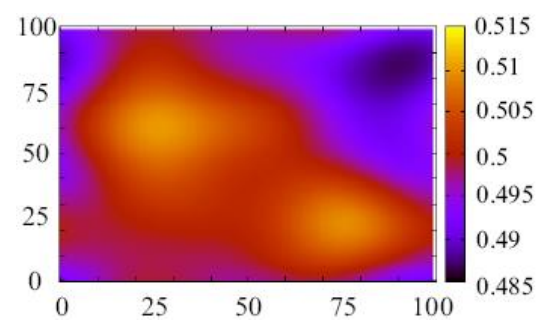

Fig. 10. Initial conditions (1).
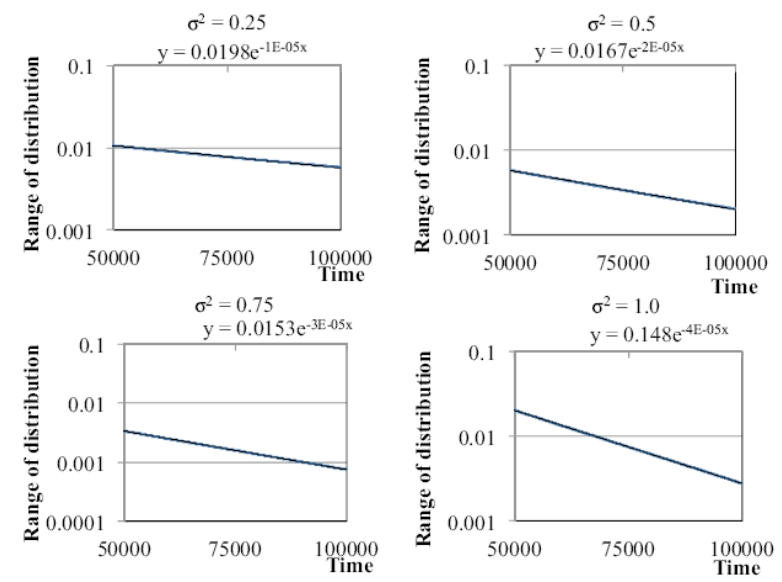

Fig. 11. Temporal variation of the distribution range due to diffusion.

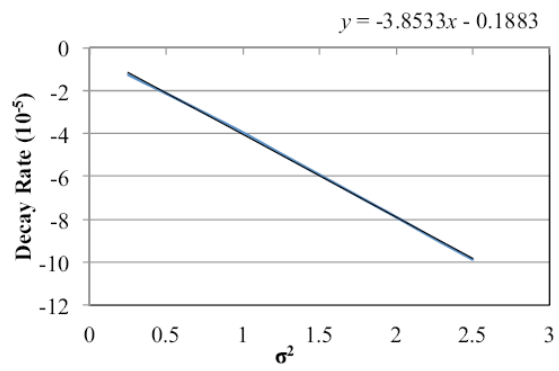

Fig. 12. Damping characteristics in the distribution range due to diffusion.

TABLE I: EXPERIMENTAL PARAMETERS (DIFFUSION ONLY)

\begin{tabular}{|c|c|c|}
\hline $\mathrm{c}$ & $\sigma^{2}$ & $\gamma$ \\
\hline 0.01 & varied & 0.1 \\
\hline
\end{tabular}

Fig. 11 shows the temporal variation of the range of the distribution due to the diffusion effect (without the drift effect). This figure is a semilog plot, and the unit of time is the number of steps. Each node computes its own distribution at every step. The parameters used are listed in Table I. It is seen that exponential decay occurs regardless of the value of $\sigma^{2}$. The relationship of $\sigma^{2}$ and the decay rate is shown in Fig. 12; $\sigma^{2}$ is proportional to the decay rate of the diffusion effect.

Fig. 13 is a representation of the temporal variation of the range of the distribution due to the diffusion and drift effects. The parameters used in the simulation are $c \sigma^{2}=$ 0.01 and $\gamma=0.1 ; c$ is varied. This figure is a semilog plot, and the unit of time is the number of steps. We can see from this figure that exponential decay occurs regardless of the value of $c$. Fig. 14 shows the relation of the decay rate and $c$. From these results, $c$ is proportional to the decay rate for the diffusion and drift effects.
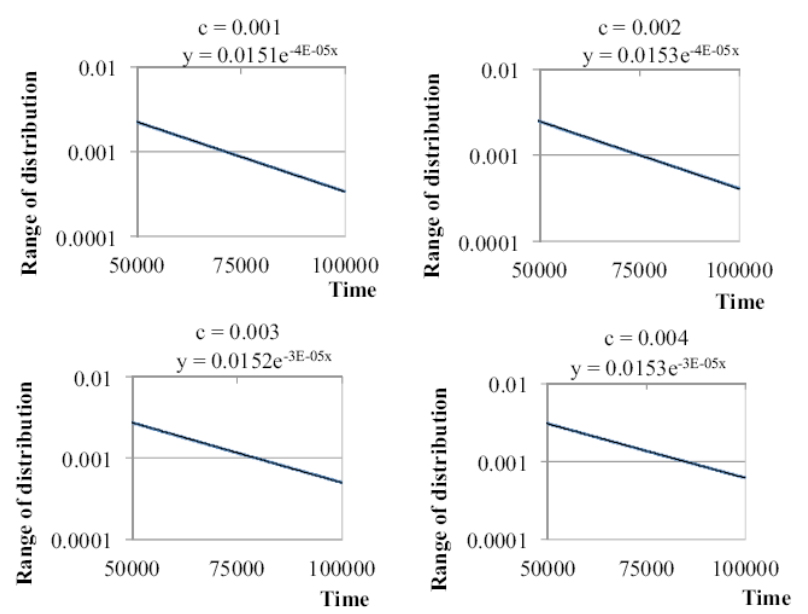

Fig. 13. Temporal variation of the distribution range due to diffusion and drift.

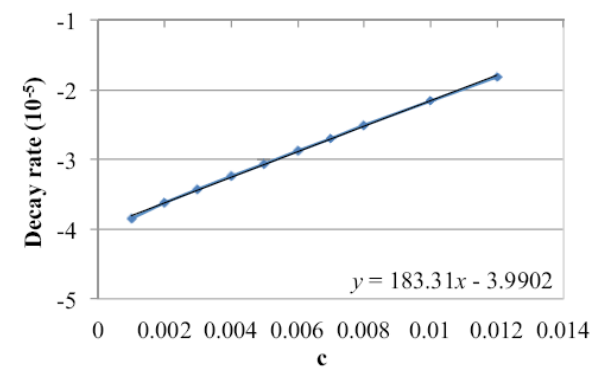

Fig. 14. Relation of the decay rate and $c$ in the distribution range due to diffusion and drift.

As mentioned above, the range of the distribution exponentially decays with time and will become zero within finite time, that is to say that it does not satisfy the asymptotic stability shown in Eq. (14).

Next, we evaluate the characteristics of the decay for the proposed method using the vector process in Sec. III and the existing method. We assume two situations:

- The initial distribution, $p\left(t_{k}\right)$, (ex. residual battery power) does not change over time, and all nodes are immovable (Sec. IV-B)

- The initial distribution, $p\left(t_{k}\right)$, decreases gradually by power consumption, and all nodes can move (Sec. IVC)

\section{B. Evaluation of Stability (in Two-Dimensional Lattice Model)}

We assume a two-dimensional lattice model with 100 $\times 100$ nodes in a torus topology; nodes do not move 
(fixed nodes). The $i$ th node's initial distribution (the initial battery capacity), $p_{i}(0)$, is set to a random number uniformly distributed in $[10,20]$ (Fig. 15). In this experiment, $p_{i}\left(t_{k}\right)$ does not change over time. Furthermore, nodes in the network do not move in this simulation. The parameters used are summarized in Table II, and the number of components of the vector for the proposed clustering model is $21 \quad(n=0,1, \cdots, 20)$. These parameters satisfy the conditions of Eq.(12). For these parameters, the range of the distribution is due to the diffusion effect being greater than the drift effect [10]. Each node sends control packets every $1 \mathrm{~s}(\Delta t=1 \mathrm{~s})$ and deals with the diffusion and drift processing on the basis of the information in the received packets. In our evaluation, the node sends control parameters for its adjacent nodes at the same time. Moreover, the simulation time is 1:0 hour.

TABLE II: EXPERIMENTAL PARAMETERS (THE TWO-DIMENSIONAL Network $\boldsymbol{Q}$ Does Not Change OVer Time)

\begin{tabular}{|c|c|c|}
\hline $\mathrm{c}$ & $\sigma^{2}$ & $\gamma$ \\
\hline 0.001 & 200.0 & 0.0001 \\
\hline
\end{tabular}

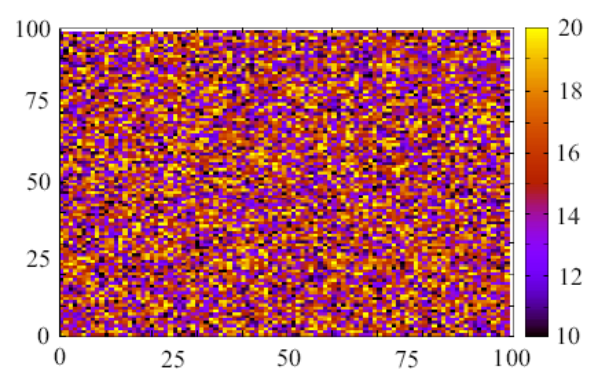

Fig. 15. Initial conditions (2).

Fig. 16 shows the cluster structures for the 5 th and 20th components of the vector at time $t_{k}\left(t_{k}=0.1,0.5,1.0\right.$ hour). We can see from this figure that the cluster of the 5th component has a finer structure than that of the 20th component. This is because the latter is strongly influenced by the diffusion effect. In other words, if we set the vector component's number to a large value, we can configure larger clusters.

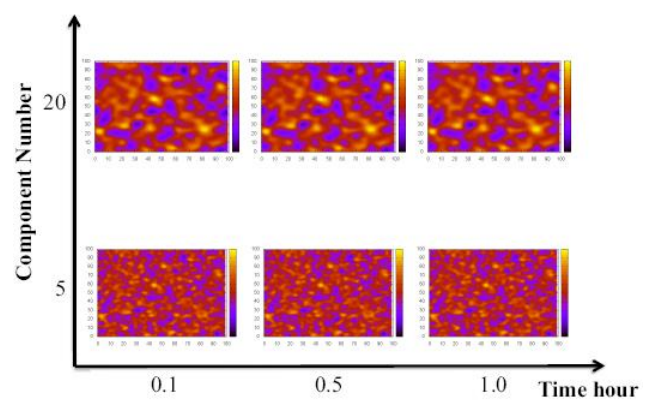

Fig. 16. Cluster structures for the 5th and 20th components of $\boldsymbol{Q}\left(t_{k}\right)$.

Fig. 17 shows the temporal variation in the distribution range for the 5 th and 20th components of $\boldsymbol{Q}\left(t_{k}\right)$. This figure includes results for the existing method [10] for comparison with our proposal. We can see from this figure that the range of the distribution for the existing method always decays, which means that the existing method cannot guarantee the stability for the range of the distribution shown in Eq. (14).

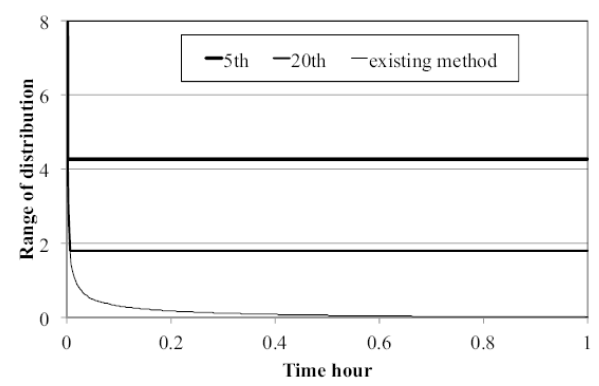

Fig. 17. Temporal variation in the distribution range for the 5 th and 20th components of $\boldsymbol{Q}\left(t_{k}\right)$.

For our proposed method, on the other hand, the range of the distribution is "completely" stable (guarantees the stability in Eq. (18)) after time $t_{n}$, which is the period of time needed to form the $n$ components of the vector structure from nothing, because the initial value of the distribution of each node does not change over time. In the case with the 5 th and 20 th components, $t_{n}=5 \times 1 \mathrm{~s}=5$ $\mathrm{s}$ and $t_{n}=20 \times 1 \mathrm{~s}=20 \mathrm{~s}$, respectively.

TABLE III: THE CONVERGED VALUE OF THE RANGE $\alpha$.

\begin{tabular}{|c|c|}
\hline 5 th & 20 th \\
\hline 4.269 & 1.804 \\
\hline
\end{tabular}

Moreover, Table III shows the converged value of the range of the distribution in each component of the vector. This result shows that the converged value of the 5 th component is greater than that of the 20th component, because the latter is strongly influenced by the diffusion effect.

\section{Evaluation of Stability (in MANET)}

In this section, we evaluate the decay characteristics of the proposed method in a network model supposing an MANET. The network model is the unit disk graph (UDG) with dimensions of $1,500 \mathrm{~m} \times 1,500 \mathrm{~m}$ and a torus topology (Fig. 18). UDG is a type of intersection graph based on circles of the same size. UDG is suitable as a model of an ad hoc network because it can describe various radio transmission ranges between nodes, but it cannot reflect more realistic wireless network characteristics such as packet collisions. We will address this issue in future work.

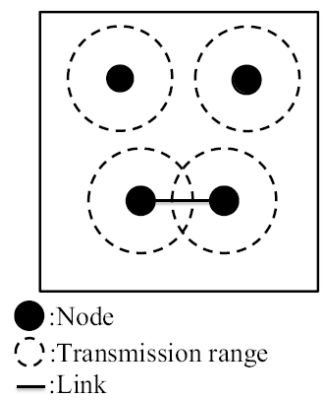

Fig. 18. Unit disc graph network. 
The simulation conditions are as follows. 100 nodes are randomly placed in the area. The initial distribution $p_{i}(0)$ for node $i$ is a uniform random number in the range of [10, 20] (Fig. 19); the radio transmission range for each node is $250 \mathrm{~m}$. Regarding mobility, all nodes move every $100 \mathrm{~s}, 10 \mathrm{~s}$, or $1 \mathrm{~s}$ in consideration of the convergence time of cluster formation. The range to which each node moves at the above intervals yields an average movement distance of $1.3 \mathrm{~m}$ (uniform random number $[0,2.6])$; the direction is based on the random direction model. The parameters of the proposed model are listed in Table IV. Note that, these parameters meet the conditions of Eq.(12). We assume that the initial distribution of each node, is identical to the random initial battery capacity, and the battery capacity of each node decreases by transmitting and processing control packets to and from neighbor nodes. Each node sends control packets every $1 \mathrm{~s}(\Delta t=1 \mathrm{~s})$ and deals with the diffusion and drift processing on the basis of the information in the received packets. Note that the node sends control packets for adjacent nodes simultaneously. Each node consumes the battery reserves at $1 \mu \mathrm{J} / \mathrm{bit}$ for the transmission and reception of packets, and that of each representative node is $0.1 \mu \mathrm{J} / \mathrm{s}$ for processing, which is self-performed [16]. In addition, the distribution $p_{i}\left(t_{k}\right)$ of node $i$ is the residual battery capacity at $t_{k}$. We assume that nodes whose residual battery capacities become zero become unavailable and secede from the network. We pay attention to the decay characteristics of the range of the cluster configured by the 5th and 20th components. Note that, in the case with the 5th and 20th components, $t_{n}=5 \times 1 \mathrm{~s}=5 \mathrm{~s}$ and $t_{n}=20 \times 1 \mathrm{~s}=20 \mathrm{~s}$, respectively.

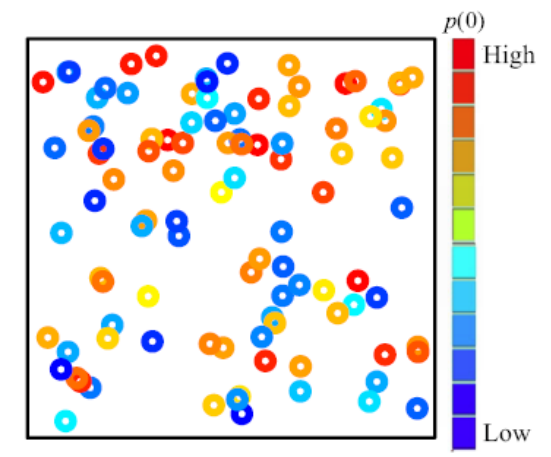

Fig. 19. Initial conditions (3).

TABLE IV: EXPERIMENTAL PARAMETERS (MANET $\boldsymbol{Q}$ CHANGES OVER TIME).

\begin{tabular}{|c|c|c|}
\hline $\mathrm{c}$ & $\sigma^{2}$ & $\gamma$ \\
\hline 0.001 & 30.0 & 0.0001 \\
\hline
\end{tabular}

Fig. 20, Fig. 21, and Fig. 22 show temporal variation in the distribution range for the 5th and 20th components for each mobility frequency. In these figures, the horizontal axis and vertical axis represent time and the range of the distribution, respectively. These results show that the range of the distribution for the existing method decreases sharply regardless of the node mobility frequency. The range for the existing method approaches zero very fast, and the existing method can not guarantee the stability in Eq. (14). On the other hand, the characteristics of the range for our proposed method maintain nearly a fixed value after time $t_{n}\left(t_{n}=5\right.$ or $\left.20 \mathrm{~s}\right)$ for all patterns of the node mobility frequency. The proposed method does not satisfy Eq. (18), but it can maintain the range of the distribution at a positive value for a long time. Table $\mathrm{V}$ summarizes the fluctuation $b$ of the range for the mobility frequency and the component number. The fluctuation $b$ is the maximum deviation between the range at time $t_{n}$ and the range at time $t\left(>t_{n}\right)$. We can see from this result that the value of $b$ becomes large as the node mobility frequency grows, and the difference between the component numbers does not influence the value of $b$. This is because a large node mobility frequency leads to drastic changes of the network topology before the formation of the clusters converges.

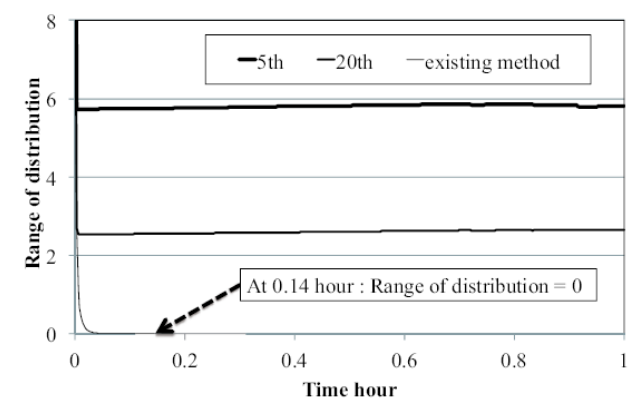

Fig. 20. Temporal variation in the range of the distribution for the 5th and 20th components of $\boldsymbol{Q}\left(t_{k}\right)$ (Nodes move every $100 \mathrm{~s}$. Node mobility frequency is low.).

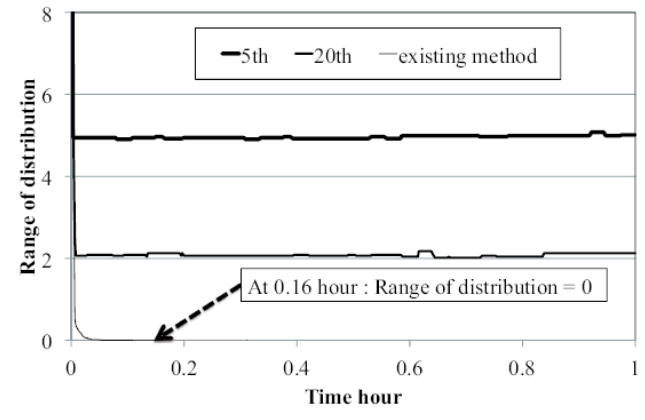

Fig. 21. Temporal variation in the range of the distribution for the 5th and 20th components of $\boldsymbol{Q}\left(t_{k}\right)$ (Nodes move every $10 \mathrm{~s}$. Node mobility frequency is moderate.).

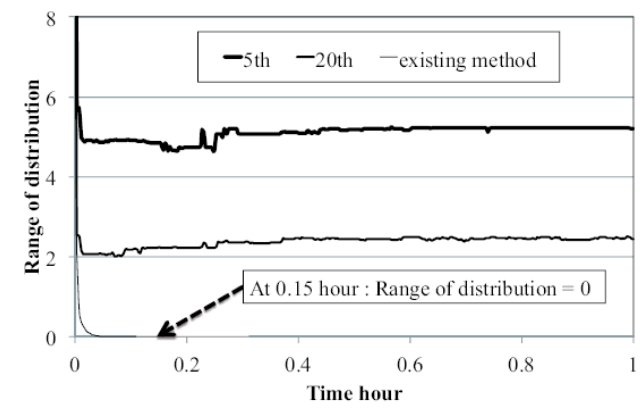

Fig. 22. Temporal variation in the range of the distribution for the 5 th and 20th components of $\boldsymbol{Q}\left(t_{k}\right)$ (Nodes move every $1 \mathrm{~s}$. Node mobility frequency is high.). 
TABLE IV: THE RANGE OF FluCtuAtion $B$

\begin{tabular}{|c|c|c|}
\hline $\begin{array}{c}\text { mobility frequency } \\
\text { (Nodes move every } f \text { s })\end{array}$ & $\begin{array}{c}\text { component } \\
\text { number } n\end{array}$ & $\begin{array}{c}\text { fluctuation } \\
b\end{array}$ \\
\hline \multirow{2}{*}{ small $(f=100)$} & 5 & 0.132 \\
\cline { 2 - 3 } & 20 & 0.124 \\
\hline \multirow{2}{*}{ moderate $(f=10)$} & 5 & 0.135 \\
\cline { 2 - 3 } & 20 & 0.128 \\
\hline \multirow{2}{*}{ large $(f=1)$} & 5 & 1.094 \\
\cline { 2 - 3 } & 20 & 0.531 \\
\hline
\end{tabular}

Next, Fig. 23, Fig. 24, and Fig. 25 show the time average of the number of clusters from $t=20 \mathrm{~s}$ to $t=3$; $600 \mathrm{~s}$ for the 5th and 20th components at each mobility frequency. The horizontal axis represents the vector component, and the vertical axis represents the number of clusters. The results show that the time average of the number of clusters yielded by the 20th component is smaller than that of the clusters formed by the 5th component. This indicates that the size of the configured cluster increases with the component number $n$. Thus, by changing the component number of the vector, our proposed clustering method cannot set strictly the number of clusters, but can decide the number of clusters to some (large or small) extent.

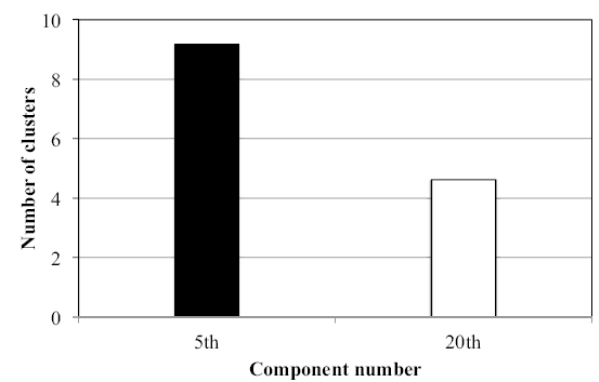

Fig. 23. Time average of the number of clusters for the 5th and 20th components of $\boldsymbol{Q}\left(t_{k}\right)$ (Nodes move every $100 \mathrm{~s}$. Node mobility frequency is low.).

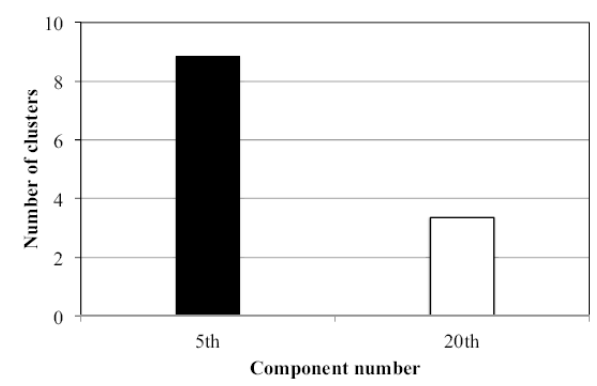

Fig. 24. Time average of the number of clusters for the 5th and 20th components of $\boldsymbol{Q}\left(t_{k}\right)$ (Nodes move every $10 \mathrm{~s}$. Node mobility frequency is moderate.).

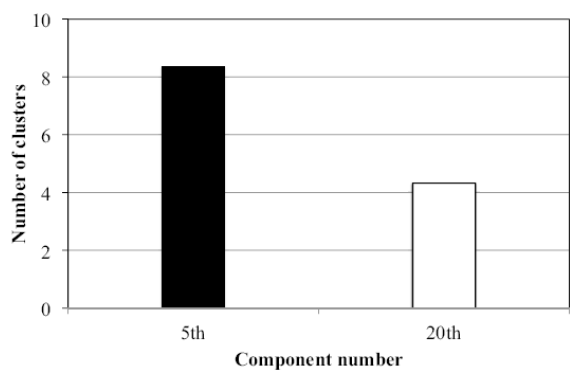

Fig. 25. Time average of the number of clusters for the 5 th and 20th components of $\boldsymbol{Q}\left(t_{k}\right)$ (Nodes move every $1 \mathrm{~s}$. Node mobility frequency is high.).

\section{CONCLUSION}

Our prior work proposed a structure formation method based on local interaction, and it was applied to an autonomous decentralized clustering method for an MANET. This method, however, the number of clusters decreases with temporal evolution. Therefore, the hierarchical management of the network cannot be performed by this problem. This study provides a solution; a method that uses distribution vectors to preserve the distribution history and thus stabilize the range of the distribution of each node. Numerical simulations clarified that our clustering mechanism can guarantee the stability of the range of the distributions formed by our proposed method. In addition, by changing the components of the distribution vector, our proposed clustering method cannot set strictly the number of clusters, but can decide the number of clusters to some (large or small) extent. The issue for our proposed method is that a certain time is required to configure clusters. As future works, it is necessary to investigate the effects of delay in a cluster configuration by the proposed method. Moreover, we should define assumptions of the target MANET clearly and reveal the influence of network protocols such as routing protocols and MAC protocols in order to use our clustering in real MANETs. Future works include that we will evaluate particularly our clustering performance considering these effects in real MANETs, and we will evaluate the performance of our clustering method considering power savings and load balancing.

\section{ACKNOWLEDGMENT}

This research was partly supported by JSPS KAKENHI Grant Numbers 26280032, 15K00431, and Project Research Grants from the Graduate School of Information Sciences, Hiroshima City University.

\section{REFERENCES}

[1] C. E. Perkins, ed., Ad Hoc Networking, Addition Wesley, 2000.

[2] J. Y. Yu and P. H. J. Chong, "A survey of clustering schemes for mobile ad hoc networks," IEEE Commun. Surv. Tutor., vol. 7, no. 1, pp. 32-48, 2005.

[3] T. Nagata, H. Oguma, and K. Yamazaki, "A sensor networking middleware for clustering similar things," in Proc. International Workshop on Smart Object Systems in Conjunction with International Conf. on Ubiquitous Computing, 2005, pp. 53-60.

[4] S. Priyankara, K. Kinoshita, H. Tode, and K. Murakami, "A clustering method for wireless sensor networks with heterogeneous node types," IEICE Trans. Commun., vol. E94-B, no. 8, pp. 2254-2264, Aug. 2011.

[5] S. Basagni, "Distributed clustering for ad hoc networks," in Proc. of International Symposium on Parallel Architectures, Algorithms and Network, 1999 s, pp. 310-315.

[6] A. D. Amis, R. Prakash, T. H. P. Vuong, and D. Huynh, "Maxmin D-cluster formation in wireless ad hoc networks," in Proc. IEEE INFOCOM 2000, 2000, vol. 1, pp. 32-41.

[7] T. Ohta, S. Inoue, Y. Kakuda, and K. Ishida, "An adaptive multihop clustering scheme for ad hoc networks with high mobility," IEICE Trans. Fundamentals, vol. E86-A, no. 7, pp. 1689-1697, 2003. 
[8] G. Neglia and G. Reina, "Evaluating activator-inhibitor mechanisms for sensors coordination," in Proc.2nd IEEE/ACM BIONETICS, Budapest, Hungary, Dec. 2007, pp. 129-133.

[9] K. Hyodo, N. Wakamiya, E. Nakaguchi, M. Murata, Y. Kubo, and K. Yanagihara, "Reaction-diffusion based autonomous control of wireless sensor networks," International J. Sensor Networks, vol. 7, no. 4, pp. 189-198, May 2010.

[10] C. Takano, M. Aida, M. Murata, and M. Imase, "Proposal for autonomous decentralized structure formation based on local interaction and back-diffusion potential," IEICE Trans. Commun., vol. E95-B, no. 5, pp. 1529-1538, 2012.

[11] C. Takano and M. Aida, "Diffusion-type autonomous decentralized flow control for end-to-end flow in high-speed networks," IEICE Trans. Commun., vol. E88-B, no. 4, pp. 15591567, Apr. 2005.

[12] M. Aida, "Using a renormalization group to create ideal hierarchical network architecture with time scale dependency," IEICE Trans. Commun., vol. E95-B, no. 5, pp. 1488-1500, May 2012.

[13] K. Takagi, Y. Sakumoto, C. Takano, and M. Aida, "On convergence rate of autonomous decentralized structure formation technology for clustering in ad hoc networks," in Proc. IEEE ICDCS 2012 Workshops (ADSN 2012), Macau, China, Jun. 2012.

[14] H. Takayama, S. Hatakeyama, and M. Aida, "Self-adjustment mechanism guaranteeing asymptotic stability of clusters formed by autonomous decentralized mechanism," Journal of Communications, vol. 9, no. 2, pp. 180-187, Feb. 2014.

[15] K. Takagi, M. Aida, C. Takano, and M. Naruse, "A proposal of new autonomous decentralized structure formation based on Huygens' principle and renormalization," in Proc. Third International Conf. on Advanced Collaborative Networks, Systems, and Applications, Nice, France, Jul. 2013.

[16] J. Hill, R. Szewczyk, A. Woo, S. Hollar, D. Culler, and K. Pister, "System architecture directions for networked sensors," in Proc. ASPLOS-IX, New York, USA, Nov. 2000, pp. 93-104.

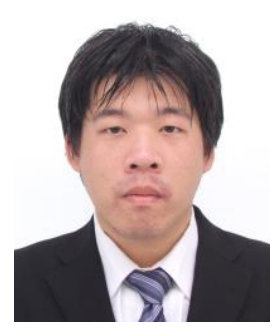

Ryo Hamamoto received the B.E. and M.E. degrees in Computer Engineering from Hiroshima City University, Japan, in 2012 and 2014, respectively. His research interests lie in the area of wireless networks and distributed systems. He received the Information Network Research Award of IEICE in 2012 and 2015. $\mathrm{He}$ is a student member of IEEE (U.S.A.) and IEICE (Japan).

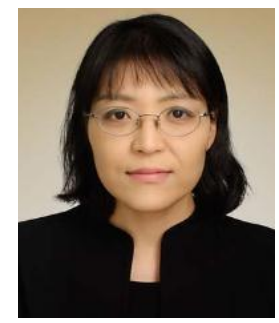

Chisa Takano received a B.E. degree in telecommunication engineering from Osaka University, Japan, in 2000, and a Ph.D. in system design engineering from Tokyo Metropolitan University, Japan, in 2008. In 2000, she joined the Traffic Research Center, NTT Advanced Technology Corporation (NTT-AT). Since April 2008, she has been an Associate Professor of the Graduate School of Information Sciences, Hiroshima City University. Her research interests lie in the area of computer networks and distributed systems. She received the IEICE Young Researchers' Award in 2003. She received the Information Network Research Award from the IEICE in 2004, 2012, and 2015. She is a member of IEEE (U.S.A.) and IEICE (Japan).

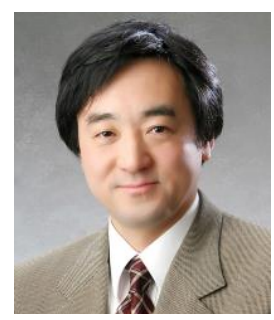

Kenji Ishida received B.E., M.Sc., and Ph.D degrees from Hiroshima University, Japan, in 1984, 1986, and 1989, respectively. He was at Hiroshima Prefectural University from 1989 to 1997. From 1997 to 2003, he was an Associate Professor at Hiroshima City University. Since 2003, he has been a Professor in the Department of Computer Engineering, Faculty of Information Sciences, Hiroshima City University. His interests include distributed computing systems and design of control procedures for computer networks. He received the Information Network Research Award of IEICE in 1998, 2000, 2012, and 2015. He is a member of IEEE (U.S.A.), ACM (U.S.A.), IEICE (Japan), and IPSJ (Japan).

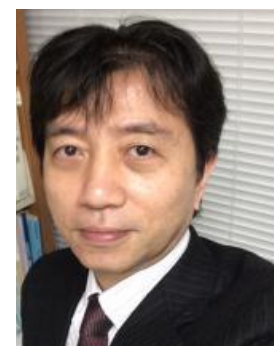

Masaki Aida received his B.S. and M.S. degrees in theoretical physics from St. Paul's University, Tokyo, Japan, in 1987 and 1989, respectively, and received a Ph.D. in telecommunications engineering from the University of Tokyo, Japan, in 1999. In April 1989, he joined NTT Laboratories. From April 2005 to March 2007, he was an Associate Professor at the Faculty of System Design, Tokyo Metropolitan University. He has been a Professor of the Graduate School of System Design, Tokyo Metropolitan University, since April 2007. His current interests include traffic issues in computer communication networks. He is a member of IEEE, IEICE, and the Operations Research Society of Japan. 